\title{
Side effects of aminoglycosides on the kidney, ear and balance in cystic fibrosis
}

\author{
Andrew Prayle, ${ }^{1}$ Alan Watson, ${ }^{2}$ Heather Fortnum, ${ }^{3}$ Alan Smyth ${ }^{1}$
}

${ }^{1}$ Child Health and Nottingham Respiratory Biomedical Research Unit, University of Nottingham, Queen's Medical Centre, Nottingham, UK ${ }^{2}$ Children's Renal and Urology Unit, Nottingham University Hospitals, Nottingham, UK ${ }^{3}$ National Biomedical Research Unit in Hearing, University of Nottingham, Nottingham, UK

\section{Correspondence to}

Alan Smyth, Child Health and Nottingham Respiratory Biomedical Research Unit, University of Nottingham, E Floor East Block, Queen's Medical Centre, Nottingham NG7 2UH, UK;

alan.smyth@nottingham.ac.uk

Received 25 November 2009 Accepted 13 March 2010

This paper is freely available online under the BMJ Journals unlocked scheme, see http:// thorax.bmi.com/site/about/ unlocked.xhtml

\section{ABSTRACT}

Aminoglycoside antibiotics are a central component of the treatment of pulmonary exacerbations of cystic fibrosis (CF) and slow the decline in lung function which ultimately causes the death of most patients. The prognosis of CF has improved, and thus side effects of treatments have become increasingly important. Observational studies suggest that the morbidity from side effects of aminoglycosides is disturbingly common, and that aggressive treatment may lead to more side effects. This review of the current literature on side effects of aminoglycosides considers the pathophysiological mechanisms, epidemiology and risk factors, investigation of side effects and preventative strategies. Treatments which have shown early promise are identified and areas of future research are discussed.

\section{INTRODUCTION}

Cystic fibrosis (CF) is the most common lifelimiting autosomal recessive disorder in the Caucasian population with approximately 7000 affected individuals in the UK. ${ }^{1}$ A mutation in the CF gene leads to a defective or absent CF transmembrane conductance regulator (CFTR) which, in most patients, ultimately leads to bronchiectasis and chronic infection with Pseudomonas aeruginosa. Intravenous aminoglycosides are used for exacerbations owing to their efficacy against $P$ aeruginosa. Patients often require several courses of intravenous antibiotics per year. The survival of patients with CF has improved, and side effects of treatment have become increasingly important.

We review the current literature on the side effects of aminoglycosides commonly used to treat pulmonary exacerbations of CF. The major side effects of aminoglycosides are kidney injury, hearing impairment and vestibular toxicity.

\section{RENAL TOXICITY IN CYSTIC FIBROSIS \\ Acute kidney injury}

Studies vary in their definition of toxicity, but approximately $5-10 \%$ of (non-CF) adult patients receiving an aminoglycoside have a significant increase in serum creatinine. ${ }^{2}$ Although toxicity is an aminoglycoside class effect, experimental data suggest that gentamicin is more toxic than tobramycin and amikacin. ${ }^{3}$ A quantitative overview of randomised controlled trials (RCTs) reached broadly the same conclusions. ${ }^{4}$

The tenacity of infection and altered pharmacokinetics in CF necessitate long courses of high-dose antibiotics. ${ }^{5}$ Since the introduction of regular 3-monthly intravenous antibiotic therapy for patients with chronic $P$ aeruginosa in Danish CF clinics in the mid-1970s, ${ }^{6}$ the management of pulmonary infection in CF has become more aggressive. However, case reports of acute kidney injury (AKI; previously termed acute renal failure) associated with aminoglycoside antibiotics in CF were not published until the late 1990s.

The first report of AKI secondary to intravenous aminoglycosides in $\mathrm{CF}^{7}$ was followed by several other cases and mini-series. Inhaled tobramycin has also been implicated. ${ }^{8}$ Bertenshaw et al ${ }^{9}$ conducted a national survey of AKI in patients with CF (AKI was pragmatically defined as an increased serum creatinine for age) and found that the incidence was 4.6-10.5/10000 CF patients/year. A subsequent case-control study ${ }^{10}$ established that an aminoglycoside in the previous week increased the risk of AKI >80-fold. Risk factors for renal impairment (including CF-related diabetes, non-steroidal antiinflammatory drugs and acute dehydration) were strongly associated with AKI (OR 24.0). Exposure to gentamicin in the previous year was associated with AKI while exposure to tobramycin was not.

\section{Chronic kidney disease}

Regular courses of aminoglycoside antibiotics may cause subclinical kidney damage leading to chronic kidney disease (CKD). This can manifest at the level of the glomerulus (causing decreased glomerular filtration rate, GFR) and the tubules (causing altered excretion of electrolytes). The stages of CKD are shown in table 1.

Pederson et a ${ }^{11}$ described a series of 46 patients aged 10-35 years who had received a mean of 20 courses of tobramycin and used $24 \mathrm{~h}$ urinary creatinine clearance to assess renal function. The cumulative dose of tobramycin was not related to the creatinine clearance; $39 \%$ of patients had reduced GFR (defined as $<96 \mathrm{ml} / \mathrm{min} / 1.73 \mathrm{~m}^{2}$ ). However, $24 \mathrm{~h}$ urinary creatinine collections are known to be unreliable, especially in children.

The largest study investigating CKD in CF was reported by Al-Aloul et al. ${ }^{12}$ Creatinine clearance was measured in $24 \mathrm{~h}$ urine collections in 80 adult patients with CF chronically infected with $P$ aeruginosa. Multiple urinary collections per patient were taken to ensure accuracy. The median number of courses of antibiotics per patient was 40 (range $1-130) ; 42 \%$ of patients had a creatinine clearance $<80 \mathrm{ml} / \mathrm{min} / 1.73 \mathrm{~m}^{2}$. Creatinine clearance declined with increasing number of antibiotic courses $(\mathrm{r}=0.59)$. Colistin was not associated with decreased kidney function when administered with a $\beta$-lactam but, when administered with an aminoglycoside, the two agents together were associated with more kidney damage than an aminoglycoside alone. Creatinine clearance can be predicted using equations such as the modification 
Table 1 Grades of chronic kidney disease categorised by glomerular filtration rate (after a table by Rees et al ${ }^{48}$ )

\begin{tabular}{ll}
\hline $\begin{array}{l}\text { Chronic kidney } \\
\text { disease grade }\end{array}$ & $\begin{array}{l}\text { Glomerular filtration } \\
\text { rate }\left(\mathbf{m l} / \mathbf{m i n} / \mathbf{1 . 7 3} \mathbf{~ m}^{2}\right)\end{array}$ \\
\hline Grade I & $>90$ \\
Grade II & $60-90$ \\
Grade III & $30-60$ \\
Grade IV & $15-30$ \\
Grade V & $<15$ \\
\hline
\end{tabular}

In general, serum biochemical anomalies may occur in grade II, symptoms appear in grade III, become increasingly severe in grade IV and renal replacement therapy is required in grade $\mathrm{V}$.

of diet in renal disease (MDRD) and Cockroft-Gault formulae. In this study, the Cockroft-Gault formula to predict creatinine clearance performed poorly and missed cases of CKD.

Investigators have used biomarkers to obtain indirect evidence of subclinical kidney injury in many research settings. $N$-acetyl- $\beta$-D-glucosaminidase (NAG), one of the best characterised biomarkers, is a proximal tubular enzyme excreted in the urine in response to kidney damage. Paediatric patients with CF administered intravenous tobramycin have increased urinary NAG levels which remain elevated weeks after treatment. ${ }^{13}$ However, patients taking inhaled gentamicin also have increased urinary NAG levels, ${ }^{14}$ suggesting that the test is highly sensitive and may not indicate clinically important renal damage.

The literature on direct comparisons of alternative intravenous agents in $\mathrm{CF}$ is complex. Comparing the relative toxicities of tobramycin and colistin in an observational study, Etherington et al $^{15}$ found that tobramycin was associated with a larger acute rise in NAG. However, increased baseline NAG levels (perhaps indicating chronic toxicity) prior to antibiotics was associated with a higher cumulative colistin dose rather than a cumulative tobramycin dose. In comparison, Al-Aloul et a $1^{12}$ found that, when administered with a non-nephrotoxic agent, colistin was cumulatively less nephrotoxic than tobramycin.

Symptomatic hypomagnesaemia associated with aminoglycosides in CF is well documented. ${ }^{16}$ Holben et al described a series of 192 patients with CF with a prevalence of hypomagnesaemia of $3 \%{ }^{17}$

\section{Mechanism of renal toxicity}

Despite extensive study, the exact mechanisms of aminoglycoside nephrotoxicity remain elusive. Care must be taken when interpreting animal models of aminoglycoside kidney injury as these studies often use doses higher than those used in clinical practice. Recent technologies allow new methods of exploring mechanisms including metabolic and transcriptome analysis, ${ }^{18}$ proteomic and microarray techniques. ${ }^{19}$

Results from gentamicin (the most extensively studied aminoglycoside) are often extrapolated to other aminoglycosides. After glomerular filtration, approximately $15 \%$ of a filtered load of an aminoglycoside is reabsorbed into the kidney. ${ }^{20}$ The uptake of aminoglycosides into the proximal tubule (a key step in the pathogenesis of kidney injury) is saturable, although the level of this effect depends upon the aminoglycoside. ${ }^{21}$ Excretion of aminoglycosides from this compartment is a slow process, taking many days. ${ }^{22}$

Testing the hypothesis that uptake of drugs into the renal cortex is saturable in humans is difficult but has been studied in a series of studies in patients scheduled for nephrectomy. ${ }^{23}$ Different dosing schedules of aminoglycosides were used and kidney cortical drug concentrations were directly measured after nephrectomy. The renal cortical concentration of amikacin and gentamicin was lower after a single dose of amikacin compared with the same dose infused over $24 \mathrm{~h}$, despite the area under the curve (AUC) of serum concentration versus time being higher. Thus, uptake of these drugs is saturable in vivo in humans. Although there was a trend to higher cortical levels when tobramycin was continuously infused, statistical significance was not achieved but the numbers of patients were limited. ${ }^{23}$

Aminoglycosides can affect the nephron at the level of the glomerulus and the tubule, although the latter has been more extensively studied. Insights into the potential mechanisms of decreased glomerular filtration have been gained from ultrastructural studies. ${ }^{24}$ Exposure of the glomerulus to gentamicin decreases the surface area available for ultrafiltration, the basement membrane increases in thickness, the glomerulus changes in shape and the number and diameter of fenestrae decrease.

The effect of gentamicin on the proximal tubular cell is summarised in figure 1. After uptake, a number of cellular processes are activated, culminating in apoptosis. This contributes to loss of the renal tubular epithelium and thus kidney dysfunction but, conversely, shedding and urinary excretion of apoptotic bodies may excrete aminoglycosides.

Changes in proximal tubular function are manifested as proteinuria, glycosuria and urinary loss of brush border enzymes. Altered tubular function has been attributed to multiple mechanisms including alteration in production of membrane transporters ${ }^{18}$ and decreased parathyroid hormone-mediated cAMP formation and subsequent decreased magnesium uptake. ${ }^{25}$

\section{Strategies to reduce nephrotoxicity of aminoglycosides}

Many groups are currently investigating strategies to reduce the nephrotoxicity of aminoglycosides. Potential approaches include blocking tubular uptake, reducing the formation of reactive oxygen species (ROS), inhibiting cellular signalling leading to apoptosis, and enhancing the ability of the kidney to regenerate. These were outlined 10 years ago but have not yet been translated into clinical practice. ${ }^{26}$

Strategies to reduce nephrotoxicity already exist. Attention to the dose prescribed and monitoring serum levels are paramount in this. Patients with CF who develop AKI are more likely to have received gentamicin in the preceding year than tobramycin when compared with controls,$^{10}$ so tobramycin is the preferred aminoglycoside in CF. Avoidance of risk factors such as concurrent non-steroidal antiinflammatory drugs and dehydration would seem prudent.

Manipulation of the dosing regimen provides a cost-effective and simple method of reducing kidney injury. Given the saturable uptake of aminoglycosides, it follows that a single daily dose would be expected to be less nephrotoxic than the same daily dose in three divided doses (see figure 2). The TOPIC study, a large randomised trial of tobramycin for patients with $\mathrm{CF}$, established that there is equal efficacy with a single daily dosing regimen as with a multiple daily dosing regimen, ${ }^{27}$ a finding confirmed in a subsequent meta-analysis. ${ }^{28}$ In the paediatric group receiving a single daily dose, the serum creatinine level decreased during the course of treatment compared with a rise in the group receiving three divided doses. In further support of renal safety, in the once daily arm the rise in NAG was $33 \%$ less than in the group receiving a three times daily regimen in both adults and children.

The pharmacokinetics of aminoglycosides are complicated by a circadian rhythm in elimination. In the once daily group of the TOPIC study, most of whom received their antibiotics in the evening, there was a lower elimination rate of tobramycin than in the three times daily group. ${ }^{29}$ There may be a diurnal variation in renal clearance of the drug, with decreased clearance occurring at night. This would lead to increased exposure of the kidneys to aminoglycoside during the course of the illness if the 
Figure 1 Potential cellular mechanism of toxicity. This diagram summarises the major themes of the literature, much of which is in vitro work and so has to be interpreted as a model. Gentamicin enters the tubular cell via the multiligand receptor megalin (shown in blue) and then uptake is via a clatharin-coated pit. Gentamicin passes in a retrograde manner via the lysosomes, Golgi and endoplasmic reticulum into the cytoplasm where it forms complexes with iron which catalyse the formation of reactive oxygen species (ROS). In the lysosomes, gentamicin causes release of the pro-apoptotic cathepsins and, in the endoplasmic reticulum, gentamicin causes release of the pro-apoptotic stress protein von Hippel-Lindau binding protein 1 (VBP1). Cytosolic gentamicin either directly or indirectly (via ROS) causes the release of cytochrome $\mathrm{C}$ from the mitochondria, a key step in apoptosis. ROS cause an increase in the gene expression of oxidative stress-inducible genes, stress-inducible chaperones and oxido-reductive enzymes. Gentamicin may have intracellular effects without entry to the cell. It can cause a rapid increase in intracellular $\mathrm{Ca}^{2+}$ and an early increase in the anti-apoptosis and pro-proliferative signals Akt and Erk; this may be mediated by the membrane $\mathrm{Ca}^{2+}$ receptor $\mathrm{CaR}$ (shown in purple).

drug is administered at night compared with the morning. This hypothesis requires testing in clinical trials.

\section{OTOTOXICITY}

\section{Ototoxicity in patients with CF}

Estimates of the prevalence of ototoxicity in patients with CF depend on the methods used and definitions of hearing impairment. The most straightforward measurement of hearing impairment is the pure tone audiogram (PTA). In this, the patient listens to a series of different frequencies and the quietest sound level at each frequency which can be reliably heard is established (the 'threshold' level, measured in decibels (dB)).

A selection of the numerous studies reporting PTAs in CF which clearly report the population studied, the definition of hearing impairment and the sample prevalence is given in table 2. A frequently cited report of the prevalence of hearing impairment is that of Mulheran et al. ${ }^{30}$ They studied 70 patients recruited from a CF clinic and, using the definition of $\geq 2$ thresholds of $>20 \mathrm{~dB}$ or one of $>25 \mathrm{~dB}$, they found an overall prevalence of hearing impairment of $17 \%$.

Oto-acoustic emissions (OAE; sounds generated by the cochlea during hearing) and distortion product OAEs (DPOAE; in which the ear generates a sound of a third pitch when presented simultaneously with two sounds of differing pitch) have been proposed as an early screening tool for cochlear damage. Patients with CF can have a normal PTA but abnormal oto-acoustic emissions which may be an indicator of early toxicity. ${ }^{31}$

Several DPOAE parameters can be measured including amplitude (volume) and latency (the amount of time for a pair of tones to travel from the ear canal to the cochlea, generate a DPOAE and return this tone back to the ear canal). The numerous methods of calculating the latency complicate the literature. Patients with CF exposed to high cumulative doses of tobramycin have alterations in latencies. ${ }^{32}$ The relevance of these findings (eg, early detection of ototoxicity) has yet to be fully understood.

\section{Mechanism of ototoxicity}

While the mechanisms of otoxicity and nephrotoxicity are similar, there are several key differences. Ototoxicity and nephrotoxicity are separate events, so patients with ototoxicity need not have nephrotoxicity and vice versa. Megalin is responsible for the uptake of aminoglycosides into the proximal tubular cells but is not found in the hair cells of the organ of Corti. ${ }^{33}$ After aminoglycoside administration, the concentration in the hair cells of the organ of Corti is static over several weeks (the kidney gradually excretes it). ${ }^{34}$

Aminoglycoside ototoxicity causes degeneration of hair cells in the organ of Corti, ${ }^{35}$ predominantly in the basal turn which is required to sense high-frequency sounds. Extracellular signalling normally causes influx of calcium into hair cells. After gentamicin exposure, calcium influx no longer occurs in basal hair cells but persists in apical hair cells. ${ }^{36}$ Thus, a clear relationship exists between the clinical features of high-frequency hearing loss, pathophysiological and histological findings.

As in the kidney, interactions between iron and aminoglycosides leading to the formation of ROS play a role in toxicity Administering iron in animal models increases ototoxicity. ${ }^{37}$ Gentamicin causes upregulation of intermediaries in the apoptosis signalling pathways between the cell surface and the nucleus. Blocking these and other intermediaries in the apoptosis cascade causes reduced apoptosis in response to gentamicin. ${ }^{38}$

Several mitochondrial ribosomal mutations are associated with ototoxicity. The most well known is the A1555G mutation in the MT-RNR1 mitochondrial gene described by Prezant et al. ${ }^{39}$ 


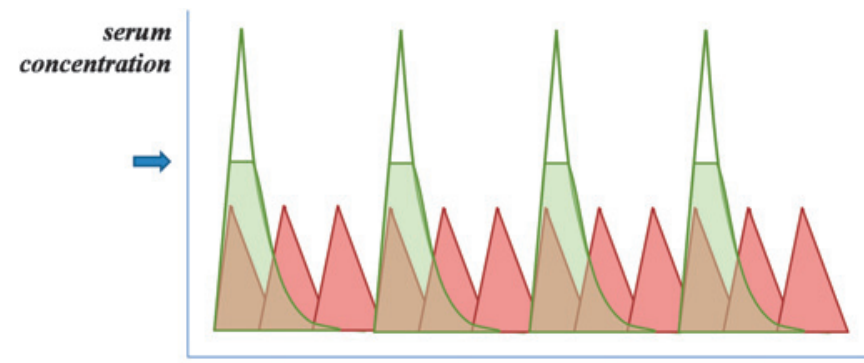

renal cortical concentration

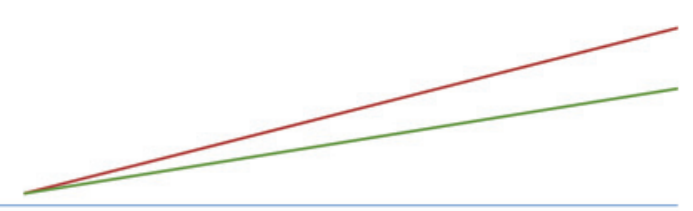

time

Figure 2 Effects of saturable uptake on kidney cortical accumulation in single daily versus three times daily dosing. In three times daily dosing (shown in red), all of the area under the curve (AUC; red shaded area) contributes to the uptake of the aminoglycoside into the kidney cortex. However, above a certain threshold (indicated by the arrow), the receptormediated uptake of the aminoglycoside is saturated and serum concentrations above this level do not contribute to the uptake of aminoglycoside into the renal cortex. As this threshold is exceeded in single daily dosing (shown in green), a proportion of the single daily dose AUC does not contribute to renal uptake. Thus, the area below this threshold contributes to renal uptake (the green area in single daily dosing). As the green area is smaller than the red area, the renal uptake in single daily dosing is thought to be lower than with the three times daily dosing regimen.

This confers a high risk of ototoxicity when an individual with the mutation is exposed to aminoglycosides. Conrad et al ${ }^{40}$ studied an unselected group of 153 adults with CF; $42.2 \%$ were found to have "mild" ototoxicity and $8.6 \%$ had "moderate to severe ototoxicity". The investigators sequenced portions of the MT-RNR1 gene of all the patients and found five with polymorphisms previously proposed as mutations associated with hearing impairment; three of these patients had hearing impairment, which was severe in two cases. They also identified variation in 16 other positions, five of whom had hearing loss.

The cost-effectiveness of screening for the A1555G mutation in patients with CF has been modelled. ${ }^{41}$ Making the assumptions of a low prevalence of the mutation $(0.086 \%)$, a high false positive screening rate, and that clinicians would switch antibiotic therapy to a less effective agent in response to a positive screening result (thus increasing the risk of mortality), this model suggested that screening all patients with CF for A1555G was not cost-effective. However, at the time epidemiological data used for the prevalence of the mutation were less than that found in the more recent large studies of Bitner-Glindzicz et al $(0.19 \%)$ and Vandebona et al $(0.21 \%){ }^{42} 43$

\section{Strategies to prevent ototoxicity}

Measures to reduce the generation or effects of ROS due to aminoglycosides have been extensively investigated and there are several promising avenues of research. A brief report of a randomised trial of aspirin versus no aspirin for 14 days in 195 patients treated with gentamicin showed a lower incidence of hearing impairment with aspirin ( $3 \%$ vs $13 \%$; $p=0.013) .{ }^{44}$ Additionally, a randomised trial comparing $\mathrm{N}$-acetylcysteine (NAC) versus no NAC in adult patients on dialysis treated with gentamicin reported fewer cases of hearing impairment in the NAC group ( $20 \%$ vs $55 \%, p=0.022) .{ }^{45}$ Both of these preliminary results require confirmation but are nevertheless encouraging.

\section{VESTIBULOTOXICITY}

Vestibulotoxicity due to aminoglycosides is secondary to damage of the hair cells in the vestibular system and does not necessarily occur with cochleotoxicity. Early symptoms of vestibulotoxicity, often insidious in onset, include vague dizziness and feeling light-headed. These may easily go unrecognised. Partial recovery can occur but can take several months.

There is little published literature regarding the prevalence of vestibulotoxicity in CF owing to the difficulty of defining and measuring it, particularly in children. A number of possible tests are available to screen for vestibular dysfunction, many of which are technically challenging for the non-specialist. Two studies have systematically screened for vestibulotoxicity in CF. Thomsen et al reported a study of 53 patients with a mean age of 11.8 years who had received a mean of 3.2 courses of tobramycin. ${ }^{46}$ A differential caloric test was performed and, in this series, no patients had an abnormal result.

Scheenstra $\mathrm{et} \mathrm{l}^{47}$ reported a detailed investigation of 23 patients with CF in the Netherlands, $30 \%$ of whom had peripheral vestibular loss diagnosed by electronystagmography (often considered the gold standard test). Of three patients who reported symptoms which suggested horizontal vestibular function loss such as rotatory dizziness, one patient had severe bilateral vestibular dysfunction. There was no association between cumulative exposure to tobramycin and vestibulotoxicity.

Comparison of the aminoglycoside exposure of the two studies is difficult as the study by Thomsen et al reported mean courses of antibiotics while the study by Scheenstra et al reported cumulative area under the curve. It is likely that the patients in the study by Scheenstra et al had a higher cumulative exposure to tobramycin.

Table 2 Selected studies investigating the prevalence of hearing loss in cystic fibrosis (CF)

\begin{tabular}{llll}
\hline Author & Study methods & Definition of HI \\
\hline Forman-Franco et al, $1979^{49}$ & PTA using frequencies 250, 500, 1000, & Air conduction thresholds for $\geq 2$ \\
& 2000, 4000, 8000 Hz. Recruited from a CF & frequencies $>25 \mathrm{~dB}$
\end{tabular}

Pedersen et al, $1987^{11}$

Mulheran et al, $2001^{30}$

Conrad et al, $2008^{40}$ 2000, 4000, $8000 \mathrm{~Hz}$. Recruited from a CF clinic $(\mathrm{n}=80)$

PTA (frequency range $125-8000 \mathrm{~Hz}$ ). High-frequency audiogram from $4000-20000 \mathrm{~Hz}$. All patients had chronic $P$ aeruginosa $(\mathrm{n}=42)$

PTA over frequencies $250-8000 \mathrm{~Hz}$. High-frequency PTA over $10000-16000 \mathrm{~Hz}(\mathrm{n}=70)$

Annual audiometric assessment of patients at two CF clinics with PTA over frequency range $1000-8000 \mathrm{~Hz}$. DPOAE were measured over the frequency range $841-7996 \mathrm{~Hz}(\mathrm{n}=153)$
Threshold increased by $\geq 15 \mathrm{~dB}$ in one or both ears at two or more adjacent frequencies

$\geq 2$ thresholds in either ear of $\geq 20 \mathrm{~dB}$ or one frequency of $\geq 25 \mathrm{~dB}$ over the frequency range $250-8000 \mathrm{~Hz}$

Either one PTA threshold $>25 \mathrm{~dB}$ or abnormal DPOAE thresholds
2/42 patients (5\%) had high-frequency $\mathrm{HI}$. Both patients had normal thresholds $<8000 \mathrm{~Hz}$

$17 \%$ had $\mathrm{HI}$

$50.8 \%$ had abnormal hearing 


\section{CONCLUSION}

Patients with CF have a large lifetime exposure to multiple medications. Aggressive treatment with intravenous antibiotics slows the progression of lung disease, decreases morbidity and delays mortality, with a cost of an increased risk of side effects. Minimising the risk of side effects is of paramount importance within the 'first, do no harm' principle.

Recent single-centre studies have suggested that the prevalence of side effects such as chronic kidney disease, ototoxicity and vestibulotoxicity are disturbingly high in the CF population. If these findings are confirmed, clinicians will need strategies to minimise the risk of toxicity. CKD has implications for lung transplantation as it is well recognised that further nephrotoxicity occurs after transplantation.

Currently, there are limited options for monitoring the early signs of toxicity. Serum creatinine-based formulae are poor at predicting renal function in the CF population; a biomarker which reliably predicts GFR (or screens patients for renal impairment) is urgently needed in the clinical setting. Similarly, once the PTA has become abnormal due to aminoglycoside ototoxicity, hearing is not expected to improve. Further research evaluating the role of DPOAEs as an early marker of cochlear damage is required.

Some strategies have already been widely adopted, such as once daily aminoglycosides, the use of tobramycin rather than gentamicin and careful monitoring of drug levels. Other approaches such as the optimum time of day of administration of antibiotics, screening for mutations which predispose to ototoxicity and the use of aspirin and NAC in patients with CF require careful consideration and further research.

\section{Competing interests None.}

Contributors All authors contributed significantly to the intellectual content of the manuscript. AS identified the need for the review. AP performed the literature search and produced the first draft. AW, HF and AS revised the manuscript and contributed extensively to subsequent drafts. All authors have approved the final version.

Provenance and peer review Not commissioned; externally peer reviewed.

\section{REFERENCES}

1. Davies JC, Alton EWFW, Bush A. Cystic fibrosis. BMJ 2007;335:1255-9.

2. Meyer RD. Risk factors and comparisons of clinical nephrotoxicity of aminoglycosides. Am J Med 1986;80:119-25.

3. De Broe ME, Giuliano RA, Verpooten GA. Choice of drug and dosage regimen. Two important risk factors for aminoglycoside nephrotoxicity. Am J Med 1986;80:115-18.

4. Buring JE, Evans DA, Mayrent SL, et al. Randomized trials of aminoglycoside antibiotics: quantitative overview. Rev Infect Dis 1988;10:951-7.

5. Touw DJ. Clinical pharmacokinetics of antimicrobial drugs in cystic fibrosis. Pharm World Sci 1998:20:149-60.

6. Frederiksen B, Lanng S, Koch C, et al. Improved survival in the Danish centertreated cystic fibrosis patients: results of aggressive treatment. Pediatr Pulmonol 1996;21:153-8

7. Samaniego-Picota MD, Whelton A. Aminoglycoside-induced nephrotoxicty in cystic fibrosis: a case presentation and review of the literature. Am J Ther 1996:3:248.

8. Hoffmann IM, Rubin BK, Iskandar SS, et al. Acute renal failure in cystic fibrosis: association with inhaled tobramycin therapy. Pediatr Pulmonol 2002;34:375-7.

9. Bertenshaw C, Watson AR, Lewis $S$, et al. Survey of acute renal failure in patients with cystic fibrosis in the UK. Thorax 2007:62:541-5.

10. Smyth A, Lewis S, Bertenshaw C, et al. Case-control study of acute renal failure in patients with cystic fibrosis in the UK. Thorax 2008:63:532-5.

11. Pedersen SS, Jensen T, Osterhammel D, et al. Cumulative and acute toxicity of repeated high-dose tobramycin treatment in cystic fibrosis. Antimicrob Agents Chemother 1987:31:594-9.

12. Al-Aloul M, Miller H, Alapati $\mathrm{S}$, et al. Renal impairment in cystic fibrosis patients due to repeated intravenous aminoglycoside use. Pediatr Pulmonol 2005;39:15-20.

13. Glass S, Plant ND, Spencer DA. The effects of intravenous tobramycin on renal tubular function in children with cystic fibrosis. J Cyst Fibros 2005;4:221-5.

14. Ring $\mathbf{E}$, Eber $\mathrm{E}$, Erwa $\mathrm{W}$, et al. Urinary $\mathrm{N}$-acetyl-beta-D-glucosaminidase activity in patients with cystic fibrosis on long term gentamicin inhalation. Arch Dis Child 1998; 78:540-3

15. Etherington C, Bosomworth M, Clifton I, et al. Measurement of urinary N-acetylb-D-glucosaminidase in adult patients with cystic fibrosis: before, during and after treatment with intravenous antibiotics. J Cyst Fibros 2007:6:67-73.
16. Green CG, Doershuk CF, Stern RC. Symptomatic hypomagnesemia in cystic fibrosis J Pediatr 1985;107:425-8.

17. Holben DH, Smith AM, Wilmott RW. Aminoglycosides lower serum magnesium concentrations in patients with cystic fibrosis: a retrospective study. J Am Diet Assoc 1995:95:1152-4

18. Xu EY, Perlina A, Vu H, et al. Integrated pathway analysis of rat urine metabolic profiles and kidney transcriptomic profiles to elucidate the systems toxicology of model nephrotoxicants. Chem Res Toxicol 2008;21:1548-61.

19. Amin RP, Vickers AE, Sistare F, et al. Identification of putative gene based markers of renal toxicity. Environ Health Perspect 2004;112:465-79.

20. Contrepois A, Brion N, Garaud JJ, et al. Renal disposition of gentamicin, dibekacin tobramycin, netilmicin, and amikacin in humans. Antimicrob Agents Chemother 1985;27:520-4.

21. Giuliano RA, Verpooten GA, Verbist $L$, et al. In vivo uptake kinetics of aminoglycosides in the kidney cortex of rats. J Pharmacol Exp Ther 1986;236:470-5.

22. Schentag JJ, Lasezkay G, Cumbo TJ, et al. Accumulation pharmacokinetics of tobramycin. Antimicrob Agents Chemother 1978:13:649-56.

23. De Broe ME, Verbist L, Verpooten GA. Influence of dosage schedule on rena cortical accumulation of amikacin and tobramycin in man. J Antimicrob Chemother 1991:27(Suppl C):41-7.

24. Cojocel C, Docius N, Maita K, et al. Renal ultrastructural and biochemical injuries induced by aminoglycosides. Environ Health Perspect 1984;57:293-9.

25. Kang HS, Kerstan D, Dai L, et al. Aminoglycosides inhibit hormone-stimulated $\mathrm{Mg}^{2+}$ uptake in mouse distal convoluted tubule cells. Can J Physiol Pharmacol 2000;78:595-602.

26. Mingeot-Leclercq M-P, Tulkens PM. Aminoglycosides: nephrotoxicity. Antimicrob Agents Chemother 1999:43:1003-12.

27. Smyth A, Tan KHV, Hyman-Taylor P, et al. Once versus three-times daily regimens of tobramycin treatment for pulmonary exacerbations of cystic fibrosis-the TOPIC study: a randomised controlled trial. Lancet 2005;365:573-8.

28. Smyth AR, Tan KH. Once-daily versus multiple-daily dosing with intravenous aminoglycosides for cystic fibrosis. Cochrane Database Syst Rev 2006;(3):CD002009.

29. Touw DJ, Knox AJ, Smyth A. Population pharmacokinetics of tobramycin administered thrice daily and once daily in children and adults with cystic fibrosis. J Cyst Fibros 2007:6:327-33.

30. Mulheran M, Degg C, Burr S, et al. Occurrence and risk of cochleotoxicity in cystic fibrosis patients receiving repeated high-dose aminoglycoside therapy. Antimicrob Agents Chemother 2001;45:2502-9.

31. Mulheran M, Degg C. Comparison of distortion product OAE generation between a patient group requiring frequent gentamicin therapy and control subjects. $\mathrm{Br} J$ Audiol 1997;31:5-9.

32. Katbamna B, Homnick DN, Marks JH. Effects of chronic tobramycin treatment on distortion product otoacoustic emissions. Ear Hearing 1999:20:393.

33. Mizuta K, Saito A, Watanabe T, et al. Ultrastructural localization of megalin in the rat cochlear duct. Hear Res 1999;129:83-91.

34. Hiel H, Bennani $\mathrm{H}$, Erre JP, et al. Kinetics of gentamicin in cochlear hair cells afte chronic treatment. Acta Otolaryngol 1992;112:272-7.

35. Zheng $\mathbf{Y}$, Schachern PA, Sone M, et al. Aminoglycoside ototoxicity. Otol Neurotol 2001:22:266-8.

36. Tan C-T, Lee S-Y, Yao C-J, et al. Effects of gentamicin and $\mathrm{pH}$ on $\left[\mathrm{Ca}^{2+}\right] \mathrm{i}$ in apica and basal outer hair cells from guinea pigs. Hear Res 2001:154:81-7.

37. Conlon BJ, Smith DW. Supplemental iron exacerbates aminoglycoside ototoxicity in vivo. Hear Res 1998;115:1-5.

38. Wei X, Zhao L, Liu J, et al. Minocycline prevents gentamicin-induced ototoxicity by inhibiting p38 MAP kinase phosphorylation and caspase 3 activation. Neuroscience 2005:131:513-21.

39. Prezant TR, Agapian JV, Bohlman MC, et al. Mitochondrial ribosomal RNA mutation associated with both antibiotic-induced and non-syndromic deafness. Nat Genet 1993:4:289-94.

40. Conrad DJ, Stenbit AE, Zettner EM, et al. Frequency of mitochondrial $12 \mathrm{~S}$ ribosomal RNA variants in an adult cystic fibrosis population. Pharmacogenet Genomics 2008:18:1095-102.

41. Veenstra DL, Harris J, Gibson RL, et al. Pharmacogenomic testing to prevent aminoglycoside-induced hearing loss in cystic fibrosis patients: potential impact on clinical, patient, and economic outcomes. Genet Med 2007;9:695-704.

42. Bitner-Glindzicz M, Pembrey $\mathbf{M}$, Duncan $A$, et al. Prevalence of mitochondrial 1555A $\rightarrow$ G mutation in European children. N Engl J Med 2009:360:640-2.

43. Vandebona $\mathbf{H}$, Mitchell $P$, Manwaring $N$, et al. Prevalence of mitochondrial 1555A $->\mathrm{G}$ mutation in adults of European descent. N Engl J Med 2009;360:642-4.

44. Sha SH, Oiu JH, Schacht J. Aspirin to prevent gentamicin-induced hearing loss. N Engl J Med 2006;354:1856-7.

45. Feldman L, Efrati S, Eviatar E, et al. Gentamicin-induced ototoxicity in hemodialysis patients is ameliorated by $\mathrm{N}$-acetylcysteine. Kidney Int 2007;72:359-63.

46. Thomsen J, Friis B, Jensen K, et al. Tobramycin ototoxicity. Repeated courses of high dosage treatment in children with cystic fibrosis. J Antimicrob Chemother 1979:5:257-60.

47. Scheenstra RJ, Rijntjes E, Tavy DL, et al. Vestibulotoxicity as a consequence of systemically administered tobramycin in cystic fibrosis patients. Acta Otolaryngol 2009:129:4-7.

48. Rees L, Webb NJA, Brogan PA. Chronic renal failure. In: Rees L, Webb NJA, Brogan PA, eds. Paediatric nephrology. Oxford: Oxford University Press, 2007:394-5.

49. Forman-Franco B, Abramson AL, Gorvoy JD, et al. Cystic fibrosis and hearing loss. Arch Otolaryngol 1979;105:338-42. 